\title{
Latest news on Arabidopsis brassinosteroid perception and signaling
}

\section{Janika Witthöft and Klaus Harter *}

Zentrum für Molekularbiologie der Pflanzen, Universität Tübingen, Tübingen, Germany

Edited by:

Andreas P. M. Weber, University of

Duesseldorf, Germany

\section{Reviewed by:}

Georg Groth, University of

Duesseldorf, Germany

Christian Hardtke, University of

Lausanne, Switzerland

Jan Willem Borst, Wageningen

University, Netherlands

*Correspondence:

Klaus Harter, Zentrum für Molekularbiologie der Pflanzen,

Pflanzenphysiologie, Universität Tübingen, Auf der Morgenstelle 1,

72076 Tübingen, Germany.

e-mail: klaus.harter@zmbp.

uni-tuebingen.de
Brassinosteroids (BRs) are plant hormones regulating growth and development. In interaction with other hormones, they are involved in environmental cue responses. The present model of the BR response pathway in Arabidopsis includes the perception of the hormone by the plasma membrane (PM) receptor brassinosteroid insensitive 1 (BRI1) and its heterooligomerization with the co-receptor BRI1-associated receptor kinase 1 (BAK1), followed by the activation of a signaling-cascade finally resulting in the expression of BR-responsive genes. New findings have shed light on the receptor density in the PM and on the molecular mechanism of BR perception, which includes the hormone-induced formation of a platform in the BRI1 extracellular domain for interaction with BAK1. Furthermore, new knowledge on early, BRI1-initiated signaling events at the PM-cytoplasm interface has recently been gained. In addition, a fast BR response pathway that modifies the membrane potential and the expansion of the cell wall - both crucial processes preceding cell elongation growth have been identified. In this review, these latest findings are summarized and discussed against the background of the present model of BRI1 signaling.

Keywords: brassinosteroids, BAK1, BRI1, membrane potential, cell elongation, P-ATPase, signal transduction, plasma membrane

\section{THE PRESENT MODEL OF BRASSINOSTEROID SIGNALING}

Brassinosteroids (BRs) are hormones regulating plant vegetative and reproductive growth and development and are involved in responses to many environmental cues, often in co-action with other hormones (Mandava et al., 1988; Clouse and Sasse, 1998). The present BR signaling-cascade model from the cell surface receptor brassinosteroid insensitive 1 (BRI1) to nuclear transcription factors, has been summarized in three recent reviews (Kim and Wang, 2010; Clouse, 2011; Yang et al., 2011). In short: in the absence of BR, BRI1 is maintained in an inactive state by the BRI1 kinase inhibitor 1 (BKI1). Signaling is initiated by the binding of the ligand to the extracellular domain (ECD) of BRI1, promoting both the recruitment of BAK1 and BRI1-mediated tyrosine phosphorylation of BKI1. The latter leads to dissociation of BKI1 from the plasma membrane (PM) and promotes trans-phosphorylation between BRI1 and BAK1. This process enhances the signaling capacity of the receptor complex and results in the phosphorylation of BR signaling kinase 1 (BSK1) and its release from the receptor complex. BSK1 initiates a complex cascade of signaling events, leading to the activation of the transcription factors BRI1EMS suppressor 1 (BES1) and brassinazole resistant 1 (BZR1). BES1 and BZR1 are predominantly responsible for eliciting the BR-specific responses that depend on differential gene expression (Sun et al., 2010). Whereas the signaling events downstream of BSKs appear to be well established, novel findings with respect to BR perception by BRI1, early steps in BRI1/BAK1 complex formation and initiation of signaling have emerged. Furthermore, a fast $\mathrm{BR}$ response pathway will be discussed that modifies the PM potential $\left(\mathrm{E}_{\mathrm{m}}\right)$ and the expansion of the wall, both crucial processes preceding cell elongation growth.

\section{BRASSINOSTEROID PERCEPTION BY BRI1}

Brassinosteroid insensitive 1 belongs to the large family of leucinerich repeat receptor-like kinases (LRR-RLKs). Recently Van Esse et al. (2011) estimated the absolute number of BRI1-GFP receptor molecules in the root tip and leaf epidermal cells. Although the number varies from 22,000 in the root meristem up to 130,000 in the elongation zone, the receptor density in the PM is constant at 12 receptors $\mu \mathrm{m}^{-2}$. Only the quiescent center $(6,700$ molecules), columella cells (10,800 molecules), and leaf epidermal cells have a lower density of 5 to 6 receptors $\mu \mathrm{m}^{-2}$ (quiescent center, columella) or 10 receptors $\mu \mathrm{m}^{-2}$ (leaf epidermis) (Van Esse et al., 2011). How far BRI1 density in the PM differs for further tissues is not known. However, quantitative analysis of BR-triggered wall expansion in Arabidopsis hypocotyl cells demonstrated a correlation between the relative number of BRI1-GFP molecules in the PM and the scale of the response (Elgass et al., 2010a). This indicates that the total amount of BRI1 and/or its density in the PM is of regulatory importance. These differences might also contribute to the differential competence of root and hypocotyl cells to respond to BR in wall expansion (Elgass et al., 2010b).

Brassinosteroid insensitive 1 comprises an ECD with 25 LRRs, with an island domain (ID) between LRRs 21 and 22 followed by a transmembrane domain. Its cytoplasmic domain consists of a juxtamembrane region, a kinase domain and a C-terminal extension (Kim and Wang, 2010; Clouse, 2011; Yang et al., 2011). However, until recently, it was not clear how BR binds to the ECD and by which molecular mechanism BRs cause BRI1 activation. Hothorn et al. (2011) have now been able to present the structure of the BRIl ECD in its free form and bound to brassinolide (BL). Their 
analysis has shed new light on BR perception and BRI1 activation: firstly, the BRI1 ECD does not show the anticipated horse shoe structure but forms a right-handed, highly twisted superhelix. The ID forms a small domain that folds back into the interior of the superhelix, where it interacts extensively with LRRs 13-25. $\mathrm{BL}$ binds by one molecule per BRI1 monomer at close proximity to the ID with the extensive contribution of LRRs. This interaction brings the hormone close to the PM (Figure 1). Secondly, for several reasons, the structure of the LRR superhelix is incompatible with BRI1-ECD oligomerization even in the presence of BL. This suggests that BRI1 activation is not mediated by ligand-induced homo-dimerization of the ECD or by conformational changes in preformed homodimers. Finally, BL binding induces a conformational rearrangement and fixing of the ID, where large parts of the hormone are still exposed to the solvent. These observations led to the idea that a stable protein-protein interaction platform is created within the BRI1 superhelix in the presence of ID-bound BL. Thus, BRI1 interaction with another protein via this platform may be the mechanism for BRI1 activation and initiation of signaling (Hothorn et al., 2011).

\section{ACTIVATION OF BRI1 BY INDUCED PROTEIN-PROTEIN INTERACTION}

Brassinolide binding creates a protein-protein interaction platform at the membrane-proximal region of BRI1, which might be crucial for receptor activation. Overwhelming evidence suggests that the favorite protein most likely to dock to the BL-induced platform within the BRI1 superhelix is BAK1 (Kim and Wang, 2010; Clouse, 2011; Yang et al., 2011). How BL-induced interaction with BAK1 transforms BRI1 into an active receptor was recently elucidated by Jaillais et al. (2011a): they studied the molecular basis for the gain-of-function phenotype of the Arabidopsis bak $1^{\text {elg }}$ mutant. The bak $1^{\mathrm{elg}}$ protein carries a substitution of aspartate 122 to asparagine in its ECD. This substitution enhances the affinity of bak $1^{\text {elg }}$ to BRI1 and may result, via more efficient BRI1/BAK1 receptor complex formation, in a more efficient transformation of BRI1 into an active receptor. Interestingly, this transformation and the full function of the BRI1/BAK1 receptor complex do not only require interaction of the ECDs but also association of the BRI1 and BAK1 kinase domains, as bak $1^{\text {elg }}$ cannot overcome the repression of BR signaling by overexpressed BKI1. As mentioned above, BKI1 prevents interaction between the BRI1 and BAK1 kinase domains and, thereby, inhibits the promotion of transphosphorylation between BRI1 and BAK1 (Jaillais et al., 2011b). Therefore, Jaillais et al. (2011a) propose a "double-lock" mechanism for BRI1/BAK1 hetero-dimerization and signaling initiation: in the absence of BR, BRI1 is kept in an inactive state by interaction with BKI1 at its C-terminal domain. In this state, BRI1 may form some ligand-independent homo-oligomers but does not interact with BAK1. The binding of BRs creates a BR-containing interaction platform inside the superhelix of BRI1, with high affinity to the ECD of BAK1, and in parallel triggers tyrosine phosphorylation of BKI1. This results in the release of BKI1 from the PM, which is paralleled by the recruitment of BAK1 by BRI1 via its interaction platform. The BRI1/BAK1 association promotes transphosphorylation between BRI1 and BAK1 and the full activation of the BRI1/BAK1 receptor complex (Figure 1). This "doublelock" mechanism is very attractive, as it provides robustness and

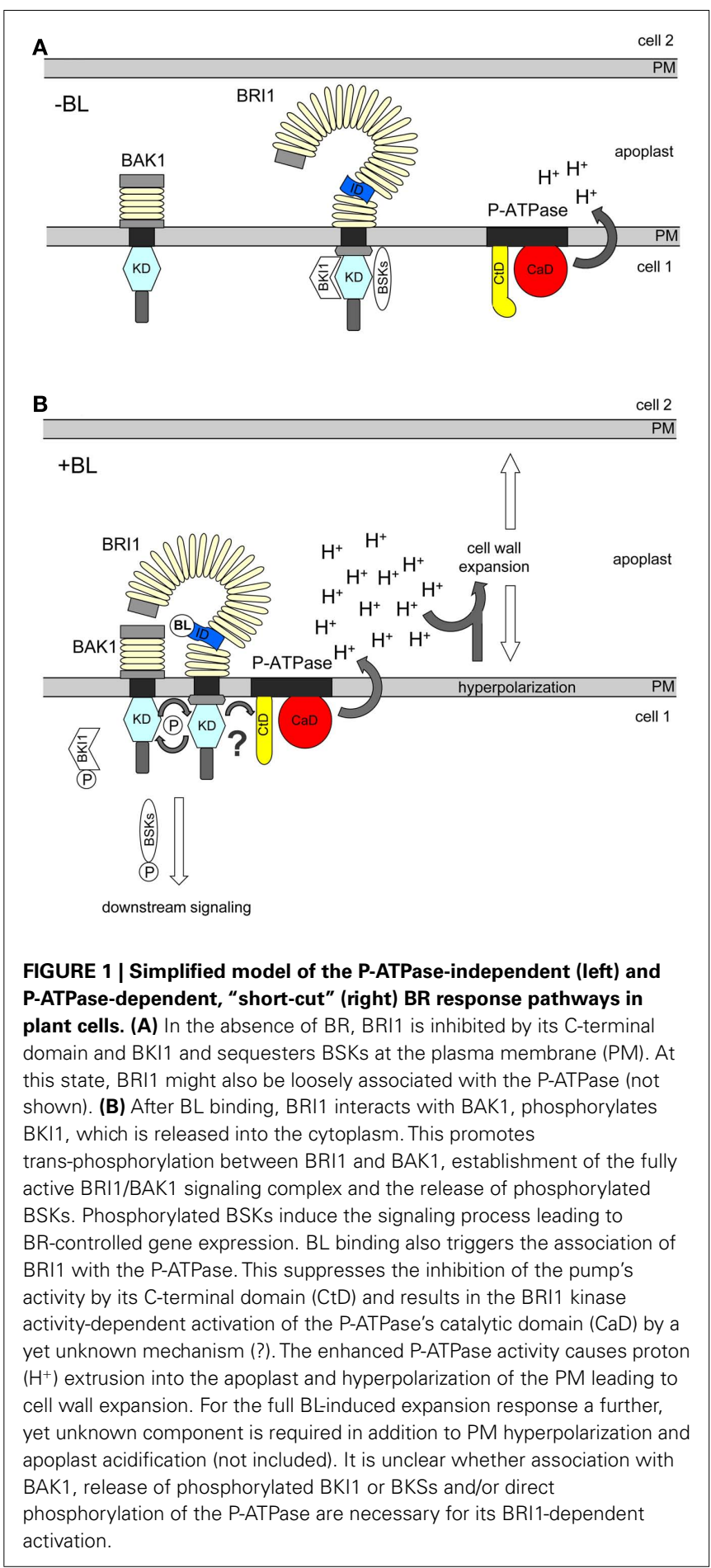

specificity for the BRI1/BAK1 complex formation and allows the regulatory modification by signals from outside and inside the cell (Jaillais et al., 2011a).

\section{A FAST BR RESPONSE PATHWAY IN THE PLASMA MEMBRANE}

Regulation of vegetative growth is a crucial BR function. Vegetative growth can be achieved either by enhanced cell elongation, 
cell number increase, or a combination of both. For instance, root length is controlled during organogenesis by components of the $\mathrm{BR}$ response pathway, including BRI1. Here, BR might determine the number of meristem cells by promoting cell cycle progression and changing the time of cell differentiation (Gonzalez-Garcia et al., 2011; Hacham et al., 2011). The effect of BL and BRI1 on elongation growth has been shown for the growth rate of BR treated epicotyl or hypocotyl isolates of bean and other plant species (Mandava, 1988; Clouse and Sasse, 1998). In Arabidopsis, BR-controlled elongation growth over several hours requires the predominantly BZR1- and BES1-mediated expression of hundreds of genes involved in water uptake, ion transport, wall, and cytoskeleton modifications (Kim and Wang, 2010; Clouse, 2011). With developmentally regulated plasma membrane polypeptide (DREPP) a novel BR-upregulated gene was identified whose product appears to be involved in the regulation of BR-mediated elongation growth at the PM-cytoplasm interface (Sun et al., 2010). Although DREPP is discussed to be involved in cytoskeleton reorganization, the molecular mechanism of DREPP elongation growth modification is not yet clear.

Brassinolide-induced elongation growth is preceded by apoplast acidification, hyperpolarization of the PM, and loosening and expansion of the cell wall (Mandava, 1988; Clouse and Sasse, 1998; Cosgrove, 2005). These processes are proposed to be regulated by the activity of PM-located P-Type ATPases (P-ATPases) such as Arabidopsis AHA1. These enzymes pump protons from the cytoplasm into the apoplast (Speth et al., 2010). However, a direct mechanistic link between activation of BRI1, up-regulation of P-ATPase activity, $\mathrm{E}_{\mathrm{m}}$ hyperpolarization, and wall loosening and expansion was not yet apparent. Recently, micro-spectroscopic methods were applied to analyze the intracellular and intramembrane dynamics, interaction pattern, physico-chemical environment and function of BRI1-GFP in living hypocotyl and root cells of transgenic Arabidopsis seedlings and transiently transformed Nicotiana benthamiana leaves (Elgass et al., 2009; Caesar et al., 2011): by measuring the dynamic distance between BRI1-GFPlabeled PMs of adjacent cells and the dimension of the wall, a BRI1dependent expansion of the wall within a few minutes after BL application was observed. In parallel, a decrease in the fluorescence lifetime (FLT) of BRI1-GFP was recorded, which points to an alteration in the receptor's close physico-chemical environment. The parameter that triggers the FLT change in BRI1-GFP in response to $\mathrm{BL}$ was identified to be the hyperpolarization of the $\mathrm{E}_{\mathrm{m}}$. By applying an inhibitor or activator of the P-ATPase, BL-regulation of the $\mathrm{E}_{\mathrm{m}}$ and expansion of the cell wall could be reversibly modulated. A quantitative analysis of the recorded data revealed that several components, which include the apoplastic $\mathrm{pH}$, the $\mathrm{E}_{\mathrm{m}}$ and yet unknown components, contribute synergistically to BL-regulated wall expansion.

Gene expression data indicate that the P-ATPase gene family members are not regulated by BRs in Arabidopsis within a few minutes after hormone application, suggesting a post-translational control of the P-ATPase activity by BRI1 to trigger $\mathrm{E}_{\mathrm{m}}$ hyperpolarization and wall expansion. One way to activate the P-ATPase activity is by BR-regulated interaction with BRI1 followed by the phosphorylation of the pump. Using different approaches, a specific interaction of BRI1 with AHA1 was demonstrated, which was modulated by BL in planta (Caesar et al., 2011). Furthermore, kinase activity of BRI1 was required for the BL-regulated change in the FLT of BRI1 and AHA1-caused $\mathrm{E}_{\mathrm{m}}$ hyperpolarization. However, the mutation of conserved threonines in the C-terminal auto-inhibitory domain of P-ATPases, which are usually required for P-ATPase activation, did not alter the interaction of mutated AHA1 with BRI1 or its capacity to induce $\mathrm{E}_{\mathrm{m}}$ hyperpolarization in the presence of activated BRI1 (Witthöft et al., 2011). Therefore, alternative mechanisms have to be discussed for how BRI1 activates the P-ATPase. In any case, these new findings suggest a "short-cut" BL-regulated signal response pathway within the PM, which is independent of gene expression (Figure 1). This pathway links BRI1 to the P-ATPase for the regulation of $\mathrm{E}_{\mathrm{m}}$ hyperpolarization and wall loosening and expansion.

The described pathway could also have "wider" consequences. In two recent papers, non-cell autonomous signals were proposed to participate in BR signaling in Arabidopsis (Scacchi et al., 2010; Hacham et al., 2011). As most plant cells are electrically coupled through plasmodesmata, they tend to respond in concert to variations in the $\mathrm{E}_{\mathrm{m}}$ (Spanswick, 1972). Although there is electrical resistance between the symplasts, it is possible that the BL-induced $\mathrm{E}_{\mathrm{m}}$ change in one cell is "communicated" to the neighboring cells. As the $\mathrm{E}_{\mathrm{m}}$ is the driving force for many transport processes, this may have significant physiological consequences for the neighboring cells (Blatt, 2004). Changes in the apoplastic pH have similar non-cell autonomous effects (Blatt and Armstrong, 1993; Blatt, 2004). Furthermore, alterations in the rigidity of the cell wall and, thus, the pressure conditions between cells, which occur during BL-induced wall loosening and expansion, may also have non-cell autonomous effects (Braybrook and Kuhlemeier, 2010).

Intriguingly, although the changes in the $\mathrm{E}_{\mathrm{m}}$ and wall dimension occur within $15 \mathrm{~min}$ after BL application (Elgass et al., 2009; Caesar et al., 2011), the onset of cell elongation is observed at the earliest after $45 \mathrm{~min}$ (see References in Clouse, 2011). Furthermore, the BL-triggered $\mathrm{E}_{\mathrm{m}}$ hyperpolarization has also been shown for tobacco epidermal leaf cells, which do not elongate (Caesar et al., 2011; Witthöft et al., 2011). This suggests that post-translational regulation of the P-ATPase activity alone is not sufficient to promote BR-triggered cell elongation growth. This probably requires the expression of genes encoding enzymes necessary for production and continuous incorporation of wall material during elongation. Alternatively, an additional growth-promoting hormone such as auxin has first to be produced by up-regulation of the corresponding biosynthetic genes. In a variety of bioassays, auxin has been shown to synergistically promote cell elongation with BRs (Halliday, 2004; Nemhauser et al., 2004; Sanchez-Rodriguez et al., 2010). Nevertheless, $\mathrm{E}_{\mathrm{m}}$ polarization and wall expansion are two excellent cell physiological readouts to elucidate the molecular details of the BR/BRI1-P-ATPase response pathway.

\section{PERSPECTIVE}

Recently published work provided new insights into BR perception by BRI1 and initiation of signaling by the active BRI1/BAK1 receptor complex at the apoplast-PM-cytoplasm interface. Furthermore, a BR response pathway has been identified that triggers cell wall expansion and $\mathrm{E}_{\mathrm{m}}$ polarization. However, there are still 
many questions to be addressed: how is BR-binding to the ECD translated into enhanced BRI1 kinase activity? What is the molecular mechanism which results in the release of BSK1? With respect to the "short-cut" pathway: are other components of the early BRI1 signaling pathway, such as BAK1 or BKI1, or elongation growth-modifying proteins, such as DREPP, involved? Which protein domain of BRI1 is responsible for interaction with the P-ATPase and is there also a function of the BR-induced BRI1 protein interaction platform? Is phosphorylation outside the $\mathrm{C}$ terminal auto-inhibitory domain required for activation of the P-ATPase and is BRI1 itself the kinase that phosphorylates the pump? What is the nature of the proposed non-cell autonomous BR signal?

Thus, though the BR response pathway is one of the best understood in plants, its further elucidation will continue to be an exciting undertaking.

\section{ACKNOWLEDGMENTS}

We thank Michael R. Blatt for helpful comments and Felicity deCourcy for proofreading the manuscript. This work was funded by the DFG grant HA2146/10-1 within the framework of the Arabidopsis Functional Genomics Network (AFGN).

\section{REFERENCES}

Blatt, M. R. (2004). Concepts and techniques in plant membrane physiology. Annu. Plant Rev. 15, 1-10.

Blatt, M. R., and Armstrong, F. (1993). $\mathrm{K}+$ channels of stomatal guard cells: abscisic-acid-evoked control of outward rectifier mediated by cytoplasmic pH. Planta 191, 330-341.

Braybrook, S. A., and Kuhlemeier, C. (2010). How a plant builds leaves. Plant Cell 22, 1006-1018.

Caesar, K., Elgass, K., Chen, Z., Huppenberger, P., Witthöft, J., Schleifenbaum, F., Blatt, M. R., Oecking, C., and Harter, K. (2011). A fast brassinolide-regulated response pathway in the plasma membrane of Arabidopsis thaliana. Plant J. 66, 528-540.

Clouse, S. D. (2011). Brassinosteroid signal transduction: from receptor kinase activation to transcriptional networks regulating plant development. Plant Cell 23, 1219-1230.

Clouse, S. D., and Sasse, J. M. (1998). Brassinosteroids: essential regulators of plant growth and development. Annu. Rev. Plant Physiol. Plant Mol. Biol. 49, 427-451.

Cosgrove, D. J. (2005). Growth of the plant cell wall. Nat. Rev. Mol. Cell Biol. 6, 850-861.

Elgass, K., Caesar, K., Schleifenbaum, F., Stierhof, Y. D., Meixner, A. J., and Harter, K. (2009). Novel application of fluorescence lifetime and fluorescence microscopy enables quantitative access to subcellular dynamics in plant cells. PLoS ONE 4, e5716. doi:10.1371/journal.pone.0005716

Elgass, K., Caesar, K., Stierhof, Y.D., Meixner, A. J., and Harter, K. (2010a). The fluorescence lifetime of BRI1-GFP as probe for the noninvasive determination of the membrane potential in living cells. SPIE Proc. 7568, 756838.
Elgass, K., Caesar, K., Wanke, D., Meixner, A. J., Harter, K., and andSchleifenbaum, F. (2010b). Application of FLIM-FIDSAM for the in vivo analysis of hormone competence of different cell types. Anal. Bioanal. Chem. 398, 1919-1925.

Gonzalez-Garcia, M. P., Vilarrasa-Blasi, J., Zhiponova, M., Divol, F., MoraGarcia, S., Russinova, E., and CanoDelgado, A. I. (2011). Brassinosteroids control meristem size by promoting cell cycle progression in Arabidopsis roots. Development 138, 849-859.

Hacham, Y., Holland, N., Butterfield, C., Ubeda-Tomas, S., Bennett, M. J., Chory, J., and Savaldi-Goldstein, S. (2011). Brassinosteroid perception in the epidermis controls root meristem size. Development 138, 839-848.

Halliday, K. J. (2004). Plant hormones: the interplay of brassinosteroids and auxin. Curr. Biol.14, R1008-R1010.

Hothorn, M., Belkhadir, Y., Dreux, M., Dabi, T., Noel, J. P., Wilson, I. A., and Chory, J. (2011). Structural basis of steroid hormone perception by the receptor kinase BRI1. Nature 474, 467-471.

Jaillais, Y., Belkhadir, Y., BalsemaoPires, E., Dangl, J. L., and Chory, J. (2011a). Extracellular leucine-rich repeats as a platform for receptor/coreceptor complex formation. Proc. Natl. Acad. Sci. U.S.A.108, 8503-8507.

Jaillais, Y., Hothorn, M., Belkhadir, Y., Dabi, T., Nimchuk, Z. L., Meyerowitz, E. M., and Chory, J. (2011b). Tyrosine phosphorylation controls brassinosteroid receptor activation by triggering membrane release of its kinase inhibitor. Genes Dev. 25, 232-237.
Kim, T. W., and Wang, Z. Y. (2010) Brassinosteroid signal transduction from receptor kinases to transcription factors. Annu. Rev. Plant Biol. 61, 681-704.

Mandava, N. B. (1988). Plant growthpromoting brassinosteroids. Annu. Rev. Plant Physiol. Plant Mol. Biol. 39, 23-52.

Nemhauser, J. L., Mockler, T. C., and Chory, J. (2004). Interdependency of brassinosteroid and auxin signaling in Arabidopsis. PLoS Biol. 2, e258. doi:10.1371/journal.pbio.0020258

Sanchez-Rodriguez, C., Rubio-Somoza, I., Sibout, R., and Persson, S. (2010). Phytohormones and the cell wall in Arabidopsis during seedling growth. Trends Plant Sci. 15, 291-301.

Scacchi, E., Salinas, P., Gujas, B., Santuari, L., Krogan, N., Ragni, L. Berleth, T., and Hardtke, C. (2010). Spatio-temporal sequence of crossregulatory events in root meristem growth. Proc. Natl. Acad. Sci. U.S.A. 107, 22734-22739.

Spanswick, R. M. (1972). Electrical coupling between cells of higher plants: a direct demonstration of intercellular communication. Planta 102 215-227.

Speth, C., Jaspert, N., Marcon, C., and Oecking, C. (2010). Regulation of the plant plasma membrane $\mathrm{H}+$ ATPase by its C-terminal domain: what do we know for sure? Eur. J. Cell Biol. 89, 145-151.

Sun, Y., Fan, X. Y., Cao, D. M., Tang, W., He, K., Zhu, J. Y., He, J. X. Bai, M. Y., Zhu, S., Oh, E., Patil, S., Kim, T. W., Ji, H., Wong, W. H., Rhee, S. Y., and Wang, Z. Y. (2010). Integration of brassinosteroid signal transduction with the transcription network for plant growth regulation in Arabidopsis. Dev. Cell 19 765-777.
Van Esse, W., Westphal, A. H., Preethi, R., Albrecht, C., van Veen, B., Borst, J. W., and de Vries, S. C. (2011). Quantification of the BRIl receptor in planta. Plant Physiol. 156, 1691-1700.

Witthöft, J., Caesar, K., Elgass, K., Huppenberger, P., Kilian, J., Schleifenbaum, F., Oecking, C., and Harter, K. (2011). The activation of the Arabidopsis P-ATPase 1 by the brassinosteroid receptor BRI1 is independent of threonine 948 phosphorylation. Plant Signal. Behav. 6, 1063-1066.

Yang, C.-J., Zhang, C., Lu, Y.-N., Jin, J.-Q., and Wang, X.-L. (2011). The mechanism of brassinosteroids' action: from signal transduction to plant development. Mol. Plant 4, 588-600.

Conflict of Interest Statement: The authors declare that the research was conducted in the absence of any commercial or financial relationships that could be construed as a potential conflict of interest.

Received: 29 August 2011; accepted: 10 September 2011; published online: 04 October 2011.

Citation: Witthöft $J$ and Harter $K$ (2011) Latest news on Arabidopsis brassinosteroid perception and signaling. Front. Plant Sci. 2:58. doi: 10.3389/fpls.2011.00058

This article was submitted to Frontiers in Plant Physiology, a specialty of Frontiers in Plant Science.

Copyright (c) 2011 Witthöft and Harter. This is an open-access article subject to a non-exclusive license between the authors and Frontiers Media SA, which permits use, distribution and reproduction in other forums, provided the original authors and source are credited and other Frontiers conditions are complied with. 